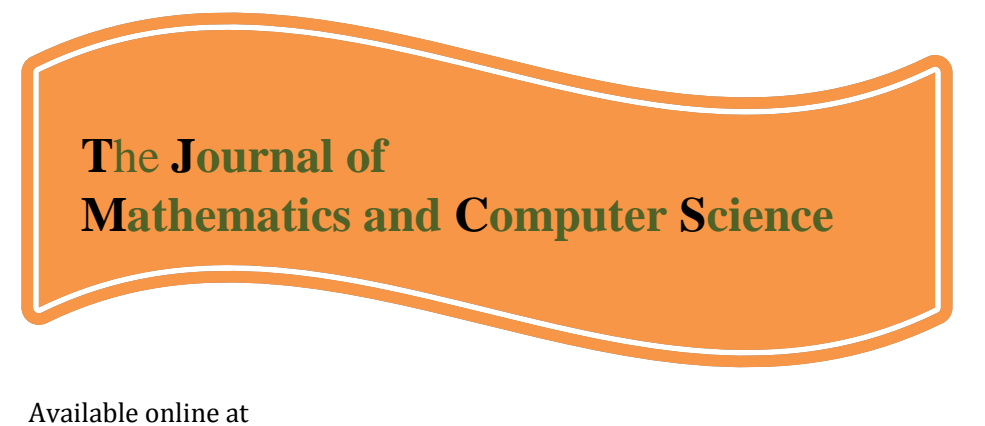

\title{
http://www.TJMCS.com
}

The Journal of Mathematics and Computer Science Vol .5 No. 1 (2012) 17-21

\section{On BP-algebras and QS-algebras}

\author{
S.A. Nematoalah Zadeh \\ PayameNoor Tehran University, Tehran, Iran \\ sanemat254@yahoo.com \\ A. Radfar \\ PayameNoor Boshehr University, Boshehr, Iran \\ ateferadfar@yahoo.com

\section{A. Borumand Saied} \\ Shahid Bahonar University of Kerman, Kerman, Iran \\ arsham@mail.uk.ac.ir
}

Received: April 2012, Revised: December 2012

Online Publication: December 2012

\begin{abstract}
In this paper we prove that the class of QS-algebras, $\mathrm{p}$-semi simple algebras and BP-algebras are equivalent.
\end{abstract}

Keywords: QS-algebra, BP-algebra, BCI-algebras.

\section{Mathematics Subject Classification: Primary 54A40; Secondary 46 S40.}

\section{Introduction.}

In 1966, Y. Imai and K. Iseki introduced two classes of abstract algebras: BCK-algebras and BCI-algebras $[5,6]$. It is known that the class of BCK-algebras is a proper subclass of BCIalgebra. P-Semisimple algebras are another special class of BCI-algebras, which were introduced by T. D. Lei in 1982 ([11]). They play a basic role in the theory of BCI-algebras and have close contacts with abelian groups. Neggers, Ahn and Kim ([12]) introduced the notion of Q-algebras in 2001 and after that Ahn and Kim introduced the notion of QSalgebras which is a generalization of Q-algebras. In 2002, Neggers and Kim [14] introduce the notion of B-algebra and obtained several results. In 2006, G. B. Kim and H.S Kim ([8]) introduced 
the notion of a BM-algebra which is a specialization of B-algebra. The concept of a BP-algebra is introduced by S. S. Ahn and J. S. Han [9], which is another generalization of B-algebra.

In this paper, we prove that QS-algebras and BP-algebras are equal to P-Semisimple algebras and so they are equal together.

\section{Preliminaries.}

Definition2.1. [8] A BM-algebra is a non-empty set X with a constant 0 and a binary operation " * " satisfying the following axioms:

(A1) $x * 0=x$,

(A2) $(z * x) *(z * y)=y * x$,

for all $x, y, z \in X$.

Definition2.2. [17] Let $X$ be a set with a binary operation * and a constant 0 . Then $(X, *, 0)$ is called BCI- algebra if satisfies the following conditions:

(BCI-1) $((x * y) *(x * z)) *(z * y)=0$,

(BCI-2) $(x *(x * y)) * y=0$,

(BCI-3) $x * x=0$,

(BCI-4) $x * y=0$ and $y * x=0$ imply $x=y$,

for all $x, y, z \in X$.

Definition2.3.[17] A BCI-algebra $X$ is called $\quad \mathrm{p}$-semi simple-algebra if $0 *(0 * x)=x$, for all $x \in X$.

Theorem 2.4. [17] Let $X$ be a BCI-algebra. Then the following hold:

(i) $x * 0=x$,

(ii) (ii) $(x * y) * z=(x * z) * y$,

for all $x, y, z \in X$.

Theorem 2.5. [17] Let X be a BCI-algebra. Then the following are equivalent:

(i) $\mathrm{X}$ is a p-semi simple algebra,

(ii) every element of $X$ is minimal,

(iii) $\quad X=\{0 * x \mid x \in X\}$.

We note that an element $x \in X$ is called minimal, if $y^{*} x=0$, implies $y=x$.

Definition 2.6.[1] Let $X$ be a set with a binary operation * and a constant 0 . Then $\left(X,{ }^{*}, 0\right)$ is called a $\mathrm{BP}$-algebra if satisfies (A2) and the following conditions:

(BP1) $x *(x * y)=y$,

(BP2) $x * x=0$.

$\mathrm{X}$ is called QS-algebra, if satisfies A1, A2, BP2 and 
(Q) $(x * y) * z=(x * z) * y$, for any $x, y, z \in X$.

Theorem 2.7.[1] Let X be a BP-algebra. Then $x * 0=x$, for all $x \in X$.

Definition 2.8. [9] A BO-algebra is an algebra $\left(X,{ }^{*}, 0\right)$ of type $(2,0)$ satisfying $A 1, B P 2$ and

$$
\text { (BO) } x *(y * z)=(x * y) *(0 * z) \text {, }
$$

for all $x, y, z \in X$.

Theorem 2.9. [9] BO-algebras, BM-algebras, $\mathrm{P}$-semi simple algebras are equivalent and they are logically equivalent by abelian group.

\section{QS-algebras, BP-algebras and P-semi simple algebras}

Theorem 3.1. Let $X$ be a set with a binary operation * and a constant 0 . Then $X$ is a p-semi simplealgebra if and only if satisfies in the following conditions:

(i) $\quad(x * y) * z=(x * z) * y$,

(ii) $\quad x * y=0$ iff $x=y$,

for all $x, y, z \in X$.

Proof. Let $X$ be a p-semi simple-algebra. Then by Theorem 2.5(ii), $x * y=0$ implies $x=y$ and by BCI-3, $x * x=0$. Hence (ii) is hold. By Theorem 2.4(ii), (i) is hold.

Conversely, let (i) and (ii) are hold. By (ii), (BCI-4) is hold. Also by (ii), $x * x=0$ and (BCI-3) is hold.

By $\quad x * x=0$ and (i) we have $\left(x^{*} 0\right) * x=(x * x) * 0=0$. Now, by (ii) and $x *(x * y)) * y=(x * y) *(x * y) * y=0$, we conclude that

$$
x *(x * y)=y \text {. }
$$

By (1), (ii) and (i) we have

$$
((x * y) *(x * z)) *(z * y)=((x *(x * z)) * y) *(z * y)=(z * y) *(z * y)=0 .
$$

Thus, $\mathrm{X}$ is a $\mathrm{BCI}$-algebra. By (ii), every element of $\mathrm{X}$ is minimal. By Theorem $2.5, \mathrm{X}$ is a p-semi simplealgebra.

Corollary 3.2. Let X be a p-semi simple-algebra. Then the following hold:

(i) $(x * y) *(x * z)=z * y$,

(ii) $x *(x * y)=y$,

for all $x, y, z \in X$.

Proof. By Theorem 3.1 and definition of p-semi simple algebra the proof is clear. 
Theorem3.3. $\mathrm{X}$ is a BP-algebra if and only if $\mathrm{X}$ is a p-semi simple-algebra.

Proof. Let X be a p-semi simple-algebra. By Theorem 3.2(i), (ii) and BCI-2, X is a BP-algebra.

Conversely, let X be a BP-algebra and $x * y=0$, for some $x, y \in X$. Then by BP1 and Theorem 2.7 $x=x * 0=x *(x * y)=y$.

Thus by BP2, $x * y=0$ if and only if $x=y$.

Let $x * y=z$, for some $x, y, z \in X$. Then by BP1, $x * z=x *(x * y)=y$. Thus $x * y=z$ if and only if $x * z=y$.

Let $(x * y) * z=t$ and $x * y=a$. By ${ }^{(2)}, x * a=y$. Thus by A2, $(x * z) * y=(x * z) *(x * a)=a * z=(x * y) * z$.

By Theorem 3.1, $X$ is a p-semi simple-algebra.

Theorem 3.4. $\mathrm{X}$ is a QS-algebra if and only if $\mathrm{X}$ is a p-semi simple-algebra.

Proof. Let $\mathrm{X}$ be a p-semi simple-algebra. Then by Theorem 3.2, $\mathrm{X}$ is a QS-algebra.

Conversely, let X be a QS-algebra. Then by A1 and A2,

$$
x *(x * y)=(x * 0) *(x * y)=y * 0=y .
$$

Thus $\mathrm{X}$ is a BP-algebra. By Theorem $3.3, \mathrm{X}$ is a p-semi simple-algebra.

Corollary 3.5. BM-algebras, BP-algebras, BO-algebras, QS-algebras and p-semisimple algebra are equivalent and they are logically equivalent by abelian group.

Proof. By Theorems 3.3, 3.4 and Theorem 2.9 the proof is clear.

\section{Conclusion.}

The concept of a BP-algebra introduced by S. S. Ahn and J. S. Han [8], which is another generalization of B-algebra. In this paper we show that this algebraic structure is equivalent to $\mathrm{p}$ semisimple BCI-algebras.

Acknowledgments: The authors would like to express their thanks to referees for their comments and suggestions which improved the paper.

\section{References}

[1] S. S. Ahn and J. S. Han, On BP-algebras, Submitted.

[2] R. Ameri, H. Hedayati, and E. Ghasemian, Filteristic soft BCK-algebras, TJMCS Vol .2 No.1 (2011) 81-87

[3] Q. P. Hu and X. Li, On BCH-algebras, Math. Seminar Notes 11 (1983), 313-320. 
S.A. Nematoalah Zadeh, A. Radfar, A. Borumand Saied/ TJ MCS Vol .5 No.1 (2012) 17-21

[4] Q. P. Hu and X. Li, On proper BCH-algebras, Math. Japonica 30 (1985), 659-661.

[5] K. Iseki and S. Tanaka, An introduction to theory of BCK-algebras, Math. Japonica 23 (1978), 126.

[6] K. Iseki, On BCI-algebras, Math. Seminar Notes 8 (1980), 125-130.

[7] Y. B. Jun, E. H. Roh and H. S. Kim, On BH-algebras, Sci. Math. Japonica Online 1 (1998), 347-354.

[8] C. B Kim and H. S. Kim, On BM-algebras, Math. Japonica, 211-215 (2006).

[9] C. B Kim and H. S. Kim, On BO-algebras, Mathematica Slovaca, 2012, Vol 62, No. 5, 855-864.

[10] H. S. Kim, Y. H. Kim and J. Neggers, Coxeters and pre-Coxeter algebras in Smarandache setting, Honam Math. J. 26, Vol. 4, 471-481, (2004).

[11] T. D. Lei, Structure of finite associative BCI-algebras\}, J. of Shaanxi Teachers Univ, 2(1982), 17 20 .

[12] J. Neggers, S. S. Ahn and H. S. Kim, On Q-algebras, Int. J. of Mathematics and Mathematical Sciences 27(no. 12), (2001), 749-757.

[13] J. Neggers and H. S. Kim, On d-algebras, Math. Slovaca 49 (1999), 19-26.

[14] J. Neggers and H. S. Kim, On B-algebras, Mate. Vesnik 54 (2002), 21-29.

[15] J. Neggers and H. S. Kim, A fundamental theorem of B-homomorphism for B-algebras, Int. Math. J. 2 (2002), 215-219.

[16] A. Walendziak, Some axiomatizations of B-algebras, Math. Slovaca 56(2006) 3, 301-306.

[17] H. Yisheng, BCI-algebra, Science Press, (2006). 